\title{
Erratum to: Detection of Dirofilaria repens and Dirofilaria immitis DNA in mosquitoes from Belarus
}

\author{
Tatiana Şuleșco $^{1} \cdot$ Tatiana Volkova $^{2} \cdot$ Svetlana Yashkova $^{3} \cdot$ Alexandru Tomazatos $^{4}$. \\ Heidrun von Thien ${ }^{4} \cdot$ Renke Lühken $^{4} \cdot$ Egbert Tannich $^{4,5}$
}

Published online: 2 June 2016

(C) Springer-Verlag Berlin Heidelberg 2016

\section{Erratum to: Parasitology Research}

DOI 10.1007/s00436-016-5118-y

The first author's last name was misspelled in the original article. Her name should read as follows: Tatiana Şuleșco.

The online version of the original article can be found at http://dx.doi.org/ 10.1007/s00436-016-5118-y.

\section{Renke Lühken}

luehken@bnitm.de

1 Institute of Zoology, Academy of Sciences of Moldova, Chisinau, Moldova

2 Scientific and Practical Center of the National Academy of Sciences of Belarus for Biological Resources, Minsk, Belarus

3 Ministry of Health of the Republic of Belarus, State Republican, Center of Hygiene, Epidemiology and Public Health, Minsk, Belarus

4 Bernhard Nocht Institute for TropicalMedicine, WHO Collaborating, Centre for Arbovirus and Haemorrhagic Fever Reference and, Research National Reference Centre for Tropical Infectious Diseases, Bernhard-Nocht-Straße 74, 20359 Hamburg, Germany

5 Centre for Infection Research (DZIF), partner site, Hamburg-Luebeck-Borstel, Hamburg, Germany 